\title{
Correlation in states of two identical particles
}

\author{
D.L. Zhou \\ Beijing National Laboratory for Condensed Matter Physics and \\ Institute of Physics, Chinese Academy of Sciences, Beijing 100080, China
}

\begin{abstract}
We identify the correlation in a state of two identical particles as the residual information beyond what is already contained in the 1-particle reduced density matrix, and propose a correlation measure based on the maximum entropy principle. We obtain the analytical results of the correlation measure, which make it computable for arbitrary two-particle states. We also show that the degrees of correlation in the same two-particle states with different particle types will decrease in the following order: bosons, fermions, and distinguishable particles.
\end{abstract}

PACS numbers: 03.65.Ud, 03.67.Mn, 89.70.Cf

Introduction. - The classification and characterization of correlations in a multi-party quantum state is a fundamental problem in quantum many particle physics and quantum information science [1, 2]. Although the theory on correlations in a state of more than two distinguishable particles is still under developing, the correlation in a state of two distinguishable particles is believed to be completely understood. A state of two distinguishable particles is uncorrelated if and only if it is a product state. The degree of correlation between the first particle and the second particle in a state of two distinguishable particles is shown to be the mutual entropy of the state [1, 3, 4]. However, these results can not be generalized directly to the case of a state for two identical particles. For example, we have no idea of what is a product state for two identical particles.

The concept of identical particles in quantum mechanics is essentially different from that in classical mechanics. Therefore the theory on correlations in a state of identical particles must consider the feature of identical particles. Particularly, identical particles in quantum mechanics are absolutely indistinguishable. Thus, for a system composed by two identical particles, the term like "the correlation between the first particle and the second particle" loses its meaning. Naturally we ask the following fundamental question: what is the correlation in a quantum state of two identical particles?

Based on the consideration of practical use of the state of identical particles as an entanglement resources, a useful approach [5, 6, 7, , 8] to solve this problem is proposed, whose basic idea is as follows. First, two distinguishable subsets of orthogonal modes are specified (here "mode" is abbreviated for "single particle state"). Then the correlation between the first subset of modes and the second subset of modes can be defined normally. Obviously, this type of correlation strongly depends on the specified subsets of modes, and therefore it is not an intrinsic property of the state.

Another instructive approach [9, 10, 11, 12] is built on the mathematical similarity of the structure of pure states between two distinguishable particles and two identical particles. As is well known, the Schmidt de- composition of a pure state of two distinguishable particles plays an important role in the characterization of its entanglement. Fortunately, the generalized Schmidt decomposition for pure states of two identical particles are discovered. The von Neaumann entropy of the normalized 1-particle reduced density matrix is suggested as an entanglement measure for a pure state of two identical particles [10].

Our correlation measure for a state of two identical particles is built on the maximum entropy principle: the least biased state on the basis of partial knowledge is the state with the maximum entropy under the constraint by the partial knowledge. This principle was first proposed to be used as the foundation of statistical mechanics by Jaynes in 1950s [13]. Remarkably, it also found important applications in characterizing different types of correlations in probability distribution of random variables 14, 15, 16] or quantum states of distinguishable particles 1, 17.

In this Letter, we propose a correlation measure for a quantum state of two identical particles based on the maximum entropy principle. Here the correlation in a state of two identical particles is identified as the residual information beyond what is already contained in the 1particle reduced density matrix.

Based on our correlation measure, we show that the degree of correlation is not only determined by the twoparticle quantum state, but also essentially influenced by the types of the two particles. Actually, the degrees of correlation in the same two-particle states with different particle types will decrease in the following order: bosons, fermions, and distinguishable particles.

Definition. - The quantum state of a system composed by two identical particles is specified by a twoparticle density matrix $\sigma^{(2)}$, whose 1-particle reduced density matrix $\sigma^{(1)}$ [18] is defined by

$$
\sigma_{\eta \tau}^{(1)} \equiv \operatorname{Tr}\left(a_{\eta} \sigma^{(1)} a_{\tau}^{\dagger}\right)=\operatorname{Tr}\left(a_{\eta} \sigma^{(2)} a_{\tau}^{\dagger}\right)
$$

where the operator $a_{\eta}$ and $a_{\tau}^{\dagger}$ are the annihilation operator of mode $\eta$ and the creation operator of mode $\tau$ respectively. The 1-particle reduced density matrix $\sigma^{(1)}$ 
tells us the average particle number in any mode for the state $\sigma^{(2)}$, and can be obtained by measuring 1-particle observables.

Now we apply the maximum entropy principle to define a correlation measure for the state $\sigma^{(2)}$ as follows. First we define a set of two-particle states with the same 1particle reduced density matrix as that of the state $\sigma^{(2)}$, i.e.,

$$
D_{1}\left(\sigma^{(2)}\right)=\left\{\rho^{(2)} \mid \rho^{(1)}=\sigma^{(1)}\right\} .
$$

Next we find out, among the states in the set $D_{1}\left(\sigma^{(2)}\right)$, the state $\sigma_{1}^{(2)}$ that takes the maximal entropy, i.e.,

$$
\sigma_{1}^{(2)}=\arg \max _{\rho^{(2)} \in D_{1}\left(\sigma^{(2)}\right)} S\left(\rho^{(2)}\right),
$$

where the von Neaumann entropy $S(\rho)=-\operatorname{Tr} \rho \ln \rho$. Then a correlation measure for the state $\sigma^{(2)}$ is defined as

$$
C_{2}\left(\sigma^{(2)}\right)=S\left(\sigma_{1}^{(2)}\right)-S\left(\sigma^{(2)}\right) .
$$

There are two essential elements in defining the above measure. One is to associate the characterization of noncorrelation property of the state $\sigma^{(2)}$ with the 1-particle reduced density matrix $\sigma^{(1)}$. The other is to realize that a state with more correlation has less entropy. Thus the correlation measure of the state $\sigma^{(2)}$ is equal to the entropy difference between the state $\sigma_{1}^{(2)}$ and the state $\sigma^{(2)}$, where the state $\sigma_{1}^{(2)}$ is the state with maximal entropy and with the same 1-particle reduced density matrix as $\sigma^{(1)}$.

Obviously, the correlation measure $C_{2}\left(\sigma_{1}^{(2)}\right)=0$, namely, the state $\sigma_{1}^{(2)}$ is a uncorrelated state of two identical particles. Thus, the exact role played by the maximum entropy principle is to provide us a mathematical tool to define the uncorrelated state of two identical particles by Eq. (3).

Analytic results. - Since the state $\sigma_{1}^{(2)}$ is determined by a constrained optimization defined by Eqs. (2) and (3), we obtain an analytic result of the state $\sigma_{1}^{(2)}$ by applying the standard Lagrange multipliers method. A simple calculation will show that

$$
\sigma_{1}^{(2)}=\exp \left(\sum_{\mu} \gamma_{\mu} \hat{n}_{\mu}\right)=\prod_{\mu} x_{\mu}^{\hat{n}_{\mu}}
$$

where the parameters $\gamma_{\mu}$ are the Lagrange multipliers in the diagonal representation, the parameters $x_{\mu}=$ $\exp \left(\gamma_{\mu}\right)$, and the total particle number $\hat{n}=\sum_{\mu} \hat{n}_{\mu}=2$. The unknown parameters $\gamma_{\mu}$ and $x_{\mu}$ are determined by the following equation

$$
\sigma_{1}^{(1)}=\sigma^{(1)}
$$

Eqs. (5) and (6) imply that the diagonal modes $\mu$ of the state $\sigma_{1}^{(2)}$ are the same as the diagonal modes of the state $\sigma^{(1)}$. Thus, in practice, we can first get the diagonal representation of the state $\sigma^{(1)}$ and determine the modes $\mu$. Then we only need to solve Eq. (6) in the diagonal representation.

All the above discussions are valid for both bosons and fermions. As is well known, there is a basic distinction between bosons and fermions. For bosons, the occupation number of a single mode can be arbitrary; while, for fermions, the occupation number of a single mode is 0 or 1 . The distinction, together with the total particle number requirement $\hat{n}=2$, makes Eq. (6) become

$$
x_{\mu} \sum_{\nu} x_{\nu} \pm x_{\mu}^{2}=\sigma_{\mu \mu}^{(1)},
$$

where the sign + is for bosons, and the sign - is for fermions.

Two direct results can be derived from the above analytical results. First, a state of two identical particles is uncorrelated if and only if it can be written in the form given by Eq. (5). Second, Eq. (77) makes the correlation measure defined by Eq. (4) become computable for arbitrary states of two identical particles.

Indistinguishable and distinguishable. - For two particles of different types, e.g., with different masses or different charges, the two particles are absolutely distinguishable. However, for two identical particles, if the modes can be divided into two subsets $\{A \alpha\}$ and $\{B \beta\}$ respectively, and $\hat{n}_{A}=\hat{n}_{B}=1$, the two identical particles become effectively distinguishable, i.e., we can call one particle as particle $A$, the other as particle $B$.

Under the condition $\hat{n}_{A}=\hat{n}_{B}=1$, it is easy to prove that the 1-particle reduced density matrix $\sigma^{(1)}$ is a twoblock diagonal matrix, with one block corresponding to particle $A$ and the other block corresponding to particle $B$, denoted by $\sigma^{(A)}$ and $\sigma^{(B)}$ respectively. Let us further denote the modes for the diagonal representation of $\sigma^{(A)}$ and $\sigma^{(B)}$ are $\{A \mu\}$ and $\{B \nu\}$. Now Eq. (6) becomes $x_{A \mu} \sum_{\nu} x_{B \nu}=\sigma_{\mu \mu}^{(A)}$, and $x_{B \nu} \sum_{\mu} x_{A \mu}=\sigma_{\nu \nu}^{(B)}$.

The normalization condition of the state $\sigma_{1}^{(2)}$ requires that $\sum_{\mu \nu} x_{A \mu} x_{B \nu}=1$. Through a few steps of calculation, as expected, we can prove that the correlation measure is equal to the mutual entropy, i.e.,

$$
C_{2}\left(\sigma^{(2)}\right)=S\left(\sigma^{(A)}\right)+S\left(\sigma^{(B)}\right)-S\left(\sigma^{(2)}\right) .
$$

In the above discussion, there is no differences for bosons and fermions, which is due to the condition $\hat{n}_{A}=\hat{n}_{B}=1$.

Pure states and Schmidt decomposition. - Correlation in pure states of two identical particles are sometimes called quantum correlation. When two identical particles becomes effectively distinguishable, it is also called quantum entanglement. For a two-particle pure state $\sigma^{(2)}$, the entropy $S\left(\sigma^{(2)}\right)=0$. 
Further more, pure states of two identical particles can always be written in the Schmidt decomposition forms, whether the two identical particles are bosons, fermions, or effectively distinguishable. These forms can be explicitly written as $\sum_{\mu} \sqrt{\sigma_{\mu \mu}^{(1)} / 2}\left|2_{\mu}\right\rangle$ for bosons, $\sum_{\mu} \sqrt{\sigma_{2 \mu 2 \mu}^{(1)}}\left|1_{2 \mu-1} 1_{2 \mu}\right\rangle$ for fermions, and $\sum_{\mu} \sqrt{\sigma_{\mu \mu}^{(A)}}\left|1_{A \mu} 1_{B \mu}\right\rangle$ for effectively distinguishable particles. In the above forms, $\sigma_{2 \mu 2 \mu}^{(1)}=\sigma_{2 \mu-12 \mu-1}^{(1)}$ for fermions, and $\sigma_{\mu \mu}^{(A)}=\sigma_{\mu \mu}^{(B)}$ for effectively distinguishable particles. The advantages of these forms are that they give directly all the information of the 1-particle reduced density matrix $\sigma^{(1)}$. Thus, for pure states of two identical particles, the correlation measure defined by Eq. (4) can be obtained by directly applying Eqs. (5) and (7).

It is easy to show that a pure two-particle state is uncorrelated if and only if the Schmidt number is 1 . In other words, for pure states, two distinguishable particles or two fermions are uncorrelated if and only if they occupy two orthogonal modes respectively, while two bosons are uncorrelated if and only if they occupy the same mode.

Correlation inequality. - In Eq. (4), the degree of correlation in a two-particle state is defined by the uncertainty decrease induced by the correlation. This definition implies that the degrees of different types of correlations are related with the same quantity, uncertainty. Thus, it is possible and reasonable to compare the degrees of different types of correlations. For example, in Ref. [17] we have shown that the degree of the total correlation in an $n$-partite quantum state is the sum of the degrees of all the irreducible $k$-party $(2 \leq k \leq n)$ correlations. Now we consider the comparison of the degrees of correlations in the states for systems composed by different types of particles.

Notice that, for any state $\sigma_{D}^{(2)}$ of two effectively distinguishable particles, there exist the counterpart states for two fermions and two bosons, which are obtained by regarding the particle indexes $\mathrm{A}$ and $\mathrm{B}$ as the indexes of the different subsets of modes. Here "the different subsets of modes" means that each mode in one subset is orthogonal to all modes in the other subset.

Let us denote these states with the same form as $\sigma_{D}^{(2)}$ for two effectively distinguishable particles, $\sigma_{F}^{(2)}$ for two fermions, and $\sigma_{B}^{(2)}$ for two bosons, respectively. It is easy to find that $S\left(\sigma_{D}^{(2)}\right)=S\left(\sigma_{F}^{(2)}\right)=S\left(\sigma_{B}^{(2)}\right)$ and $\sigma_{D}^{(1)}=$ $\sigma_{F}^{(1)}=\sigma_{B}^{(1)}$. Let us denote the basis of diagonal representation of $\sigma^{(1)}$ as $A \mu$ and $B \nu$. Then the constraints for the two particle Hilbert spaces are $\hat{n}_{A}=\hat{n}_{B}=1$ for effectively distinguishable particles, $\hat{n}_{A \mu}, \hat{n}_{B \nu}=0$ or 1 for fermions, and $\hat{n}_{A \mu}, \hat{n}_{B \nu}=0$ or 1 or 2 . Comparing these constraints, we know $D_{1}\left(\sigma_{D}^{(2)}\right) \subseteq D_{1}\left(\sigma_{F}^{(2)}\right) \subseteq D_{1}\left(\sigma_{B}^{(2)}\right)$. Note that, when comparing two states in different Hilbert spaces, we identify the state in a smaller Hilbert space with the counterpart state in a larger Hilbert space. Then the definition (3) implies that $S\left(\sigma_{D 1}^{(2)}\right) \leq S\left(\sigma_{F 1}^{(2)}\right) \leq$ $S\left(\sigma_{B 1}^{(2)}\right)$. Therefore the degrees of correlations for the three states $\sigma_{D}^{(2)}, \sigma_{F}^{(2)}$, and $\sigma_{B}^{(2)}$ defined above satisfies the inequality

$$
C_{2}\left(\sigma_{D}^{(2)}\right) \leq C_{2}\left(\sigma_{F}^{(2)}\right) \leq C_{2}\left(\sigma_{B}^{(2)}\right) .
$$

Eq. (9) tell us that, in the same quantum state, two fermions are less correlated than two bosons but more correlated than two (effectively) distinguishable particles. This result is originated from different constraints on the Hilbert spaces for different types of particles, which is explicitly demonstrated by the following typical examples.

Examples. - In order to demonstrate the power of the correlation measure, we apply it to analyze the correlations in three typical two-particle quantum states as listed in Table I. The values of the correlation measure in Table I are obtained by applying Eqs. (4), (5), and (77) for bosons and fermions, and by applying Eq. (8) for distinguishable particles respectively.

\begin{tabular}{|c|c|c|c|}
\hline Typical states & D & F & B \\
\hline$\left|1_{A 1} 1_{B 1}\right\rangle$ & 0 & 0 & $\ln 3$ \\
\hline$\frac{1}{\sqrt{2}}\left(\left|1_{A 1} 1_{B 1}\right\rangle+\left|1_{A 2} 1_{B 2}\right\rangle\right)$ & $\ln 4$ & $\ln 6$ & $\ln 10$ \\
\hline$\frac{1}{2}\left(\left|1_{A 1} 1_{B 1}\right\rangle\left\langle 1_{A 1} 1_{B 1}|+| 1_{A 2} 1_{B 2}\right\rangle\left\langle 1_{A 2} 1_{B 2}\right|\right)$ & $\ln 2$ & $\ln 3$ & $\ln 5$ \\
\hline
\end{tabular}

TABLE I: Correlation measures in typical states. Here D, F, $\mathrm{B}$ are abbreviated for distinguishable particles, fermions, and bosons respectively.

As expected, the inequality (9) is satisfied for the three states in Table I. All the values of the correlation measure for these typical states are in the form of $\ln d$ with $d$ a positive integer $(0=\ln 1)$, and the values in the second states are $\ln 2$ larger than the values in the third states. Let us explain these results as follows.

Because the reduced 1-particle reduced density matrices for the second states and the third states are the same, the maximum entropy states $\sigma_{1}^{(2)}$ must be the same for these two states. Thus the difference of the correlation measures for these two states is equal to the difference of the entropies of these two states, which is equal to $\ln 2$.

The first two states in Table I are pure states, so the correlation measures are equal to the entropy of the corresponding maximum entropy states $\sigma_{1}^{(2)}$. As is wellknown, the maximum entropy of a state in an $m$ dimensional Hilbert space is equal to $\ln m$. In fact, we find that the dimension of Hilbert space for the maximum entropy state constrained by the 1-particle reduced density matrix is $d$ exactly. It is the existence of correlation that makes the sate only occupy 1-dimensional Hilbert space. So the degree of correlation, i.e., the uncertainty decrease induced by the correlation, is $\ln d$.

Discussions and summary. - The correlation measure defined by Eqs. (14 4) has four obvious advantages. 
First, it is a universal correlation measure for all kinds of two-particle states, that is, it is valid for bosons or fermions, for indistinguishable particles or identical particles, and for pure or mixed states. Second, it gives a simple physical picture for the correlation. The degree of correlation is measured by the amount of uncertainty decrease induced by the correlation. In other words, a state with more correlation has less uncertainty, and the uncorrelated state for a two-particle state is the state with the same 1-particle state and the maximum entropy simultaneously. Third, it is computable for arbitrary twoparticle states. Four, the approach can be directly generalized to treat with the correlations in more than two identical particles, which has been made for distinguishable particles in Refs. [1, 17].

In summary, we propose a correlation measure for states of two identical particles by using the maximum entropy principle. We obtain the analytical results of the correlation measure, which make it computable for arbitrary two-particle states. Based on the correlation measure, we show that the degrees of correlation in the same two-particle states with different particle types will decrease in the following order: bosons, fermions, distinguishable particles. We hope that this informative picture for correlation is helpful for the characterization of intrinsic correlations in the system of identical particles, and can improve our understandings on its strongly correlated physics.

The author thanks B. Zeng, L. You and C.P. Sun for helpful discussions. This work is supported by NSF of China under Grant No. 10775176, and NKBRSF of China under Grant Nos. 2006CB921206 and 2006AA06Z104.
[1] N. Linden, S. Popescu, and W.K. Wootters, Phys. Rev. Lett. 89, 207901 (2002).

[2] D.L. Zhou, B. Zeng, Z. Xu, and L. You, Phys. Rev. A 74, 052110 (2006).

[3] B. Groisman, S. Popescu, and A. Winter, Phys. Rev. A 72, 032317 (2005).

[4] B. Schumacher and M.D. Westmoreland, Phys. Rev. A 74, 042305 (2006).

[5] P. Zanardi, Phys. Rev. A 65, 042101 (2002); P. Zanardi and X. Wang, J. Phys. A 35, 7947 (2002).

[6] J. R. Gittings and A. J. Fisher, Phys. Rev. A 66, 032305 (2002).

[7] H. M. Wiseman and J. A. Vaccaro, Phys. Rev. Lett. 91, 097902 (2003).

[8] Y. Shi, Phys. Rev. A 67, 024301 (2003).

[9] J. Schliemann, J. I. Cirac, M. Kus, M. Lewenstein, and D. Loss, Phys. Rev. A 64, 022303 (2001).

[10] R. Paskauskas and L. You, Phys. Rev. A 64, 042310 (2001).

[11] Y. S. Li, B. Zeng, X. S. Liu, and G. L. Long, Phys. Rev. A 64, 054302 (2001).

[12] G.C. Ghirardi and L. Marinatto, Phys. Rev. A 70, 012109 (2004).

[13] E.T. Jaynes, Phys. Rev. 106, 620 (1957).

[14] E. Schneidman, S. Still, M.J. Berry II, and W. Bialek, Phys. Rev. Lett. 91, 238701 (2003).

[15] S. Amari, IEEE Trans. IT 47, 1701 (2001).

[16] N. Ay, E. Olbrich, N. Bertschinger, and J. Jost, A unifying framework for complexity measures of finite systems, Proceedings of ECCS'06.

[17] D.L. Zhou, arXiv:0803.2747, to be published in Phys. Rev. Lett..

[18] C.N. Yang, Rev. Mod. Phys. 34, 694 (1962). 